\title{
Sulphydryl blocker induced gastric damage is ameliorated by scavenging of free radicals
}

\author{
F Karmeli, E Okon, D Rachmilewitz
}

\begin{abstract}
Background-Sulphydryl compounds and nitric oxide are essential in maintaining gastric mucosal integrity.

Aims-To characterise the gastric damage induced by a sulphydryl blocker, to evaluate the role of nitric oxide in its pathogenesis, and to reveal its possible prevention by scavenging of free radicals. Methods-Gastritis was induced in rats by addition of iodoacetamide $(0 \cdot 1 \%)$ to the drinking water, with and without daily intragastric administration of TEMPOL. After death, the stomach was resected, washed, lesion area assessed, and mucosal inflammatory mediators, myeloperoxidase and nitric oxide synthase activities were determined.
\end{abstract}

Results-Administration of iodoacetamide induced gastric mucosal erosions present for up to two weeks. Myeloperoxidase activity was increased for up to seven days and nitric oxide synthase activity was significantly decreased for up to 14 days. Treatment for seven days with the free radical scavenger, TEMPOL, decreased by $68 \%$ the damage induced by iodoacetamide.

Conclusions-Gastric damage induced by iodoacetamide, a sulphydryl alkylator, accompanied by inhibition of nitric oxide synthase activity shows the important contribution of sulphydryl compounds and nitric oxide to the maintenance of gastric mucosal integrity. Nitric oxide donation and scavenging of free radicals may be a novel approach to prevent gastric damage.

(Gut 1996; 38: 826-831)

Keywords: nitric oxide, TEMPOL, iodoacetamide, free radicals, gastric damage.

Departments of Medicine and Pathology, Hadassah University Hospital, Mount Scopus, Hebrew University Hadassah Medical School, Jerusalem, Israel E Okon

D Rachmilewitz

Correspondence to: Professor D Rachmilewitz, Professor D Rachmil Hadassah University Hospital, Mount Scopus, PO Box 24035, Jerusalem

Accepted for publication 7 December 1995
Glutathione exists in a combination of its reduced form (GSH) and its oxidised dimer in almost all mammalian cells. GSH is a nucleophilic scavenger of superoxide and also acts as a cofactor in the GSH peroxidase mediated reduction of $\mathrm{H}_{2} \mathrm{O}_{2} \cdot{ }^{1} \mathrm{GSH}$ has an important role in maintaining mucosal integrity in the gastrointestinal tract. GSH is essential for the protection of gastric epithelial ${ }^{1}$ and chief cells ${ }^{2}$ against oxidative stress. Moreover, calcium induced gastric mucosal injury is mediated by reduction in GSH content. ${ }^{3}$ In rats, the sulphydryl ( $\mathrm{SH}$ ) blocker, iodoacetamide, was previously shown to induce diffuse gastritis ${ }^{4}$ and, also, significant colonic injury, ${ }^{5}$ indicating the important contribution of $\mathrm{SH}$ compounds to the maintenance of gastrointestinal integrity. In this study the model in iodoacetamide induced gastric damage and its possible prevention by scavenging of free radicals was evaluated. This model was further characterised macroscopically and histologically, with special focus on the involvement of inflammatory mediators and nitric oxide (NO) in its pathogenesis.

NO has an important role in maintaining gastric mucosal integrity. Inhibition of gastric NO formation decreases gastric blood flow, deprives the tissue of oxygen, and increases mucosal vulnerability to intragastric administration of irritants that mildly damage the gastric mucosa. Moreover, inhibition of NO formation also increases gastric mucosal haemodynamic changes, such as those induced by ethanol administration. ${ }^{6}$ Oxygen derived radicals and non-radical species have been implicated previously in the pathogenesis of gastric ulceration ${ }^{78}$ and TEMPOL, a free radical, which is also a potent scavenger of other free radicals, was shown by us to prevent gastric mucosal lesions in rats. ${ }^{9}$ In this study the role of gastric NO formation in iodoacetamide induced gastric damage and its possible prevention by scavenging of free radicals was evaluated.

\section{Methods}

\section{Materials}

L-arginine, L-citrulline, L-NAME, D-NAME, NADPH, valine, aminoguanidine, dithiothreitol, phenylmethylsulphonyl fluoride, EDTA, EGTA, and 4-hydroxy-2,2,6,6-tetramethylpeperidine-1-oxyl (TEMPOL) were purchased from Sigma Chemical Co, St Louis, MO, USA; Dowex AG50W-X8 (Na form) $100-200$ mesh, and TRIS base (electrophoresis grade) were purchased from Bio-Rad Labs, Richmond, CA, USA; aquasol-2 was purchased from DuPont Co/NEN Research Products, Bad Hamburg, Germany.

\section{Animals}

Male, Sprague-Dawley rats, weighing 200-250 $\mathrm{g}$ and fed ad libitum were used in all the studies.

\section{Iodoacetamide induced gastritis}

Gastritis was induced by the addition of iodoacetamide $(0 \cdot 1 \%)$ to the drinking water for a period of 1-14 days. Control rats were provided with tap water without iodoacetamide. In several experiments, in addition to 


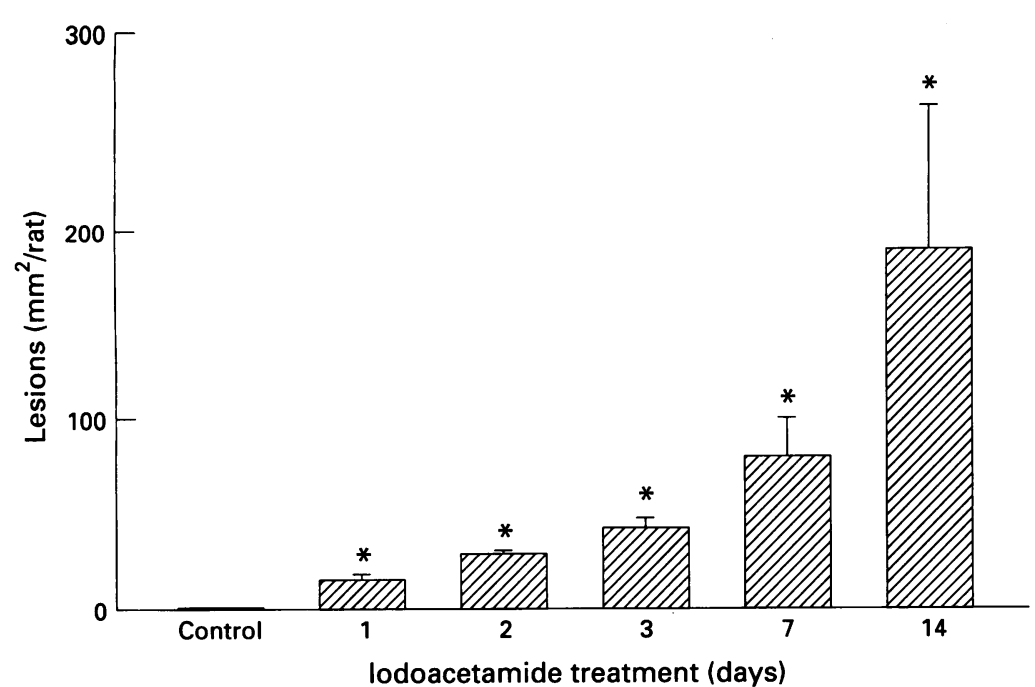

Figure 1: Gastric lesion area in iodoacetamide treated rats. Rats were treated with iodoacetamide $(0.1 \%)$ added to the drinking water. Rats were killed after 1-14 days, the stomach was removed, washed, lesion area assessed, as described in Methods. Results are mean (SEM) of 4-20 rats in each time interval. *Significantly different from control, $p<0.05$.

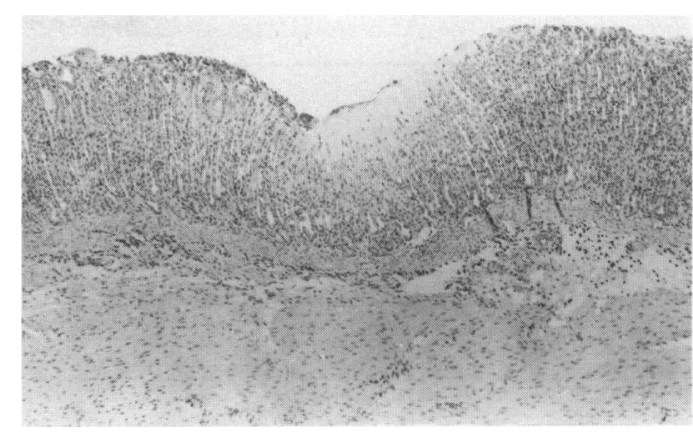

Figure 3: Histological section from the stomach of a rat treated for seven days with iodoacetamide $(0 \cdot 1 \%)$ added to the drinking water. Note the deep ulceration involving half of the mucosal thickness. Haematoxylin and eosin $\times 73$.

at $4^{\circ} \mathrm{C}$ with a polytron (Kinematica $\mathrm{GmbH}$, Kriens-Luzern, Switzerland) in $1.0 \mathrm{ml}$ of ice cold $0.5 \%$ hexadecyltrimethylammonium bromide in $50 \mathrm{mM}$ phosphate buffer, $\mathrm{pH} 6 \cdot 0$. The polytron probe was rinsed twice with $1 \cdot 0$ $\mathrm{ml}$ of the buffer and the washings were added to the homogenate. The homogenate was then sonicated for 10 seconds, freeze thawed three times, and centrifuged for 15 minutes at $40000 \mathrm{~g}$. An aliquot of the supernatant was taken for determination of the enzyme activity, according to Bradley et al. ${ }^{10}$ TEMPOL $(0.5 \mathrm{~g} / \mathrm{kg})$ intragastrically, once until death after two or seven days. Rats were killed one, two, three, seven, and 14 days after iodoacetamide treatment. The stomach was isolated, removed, washed with ice cold saline, and the extent of haemorrhagic erosions was blindly assessed. Tissue sections were obtained for histological examination and the remaining mucosa was scraped and processed for determination of lipoxygenase products, prostaglandin $\mathrm{E}_{2}\left(\mathrm{PGE}_{2}\right)$, myeloperoxidase (MPO), and NO synthase (NOS) activities.

\section{Determination of mucosal damage}

Mucosal damage was measured macroscopically and scored by multiplying the length ( $\mathrm{mm}$ ) and width $(\mathrm{mm})$. For each rat the total score was determined by summing the ulcer scores $\left(\mathrm{mm}^{2}\right)$. All measurements of damage were blindly performed by two observers using a stereomicroscope. The variability between the two observers was $7 \%$.

\section{Determination of MPO activity}

Two hundred mg of gastric mucosal scrapings were homogenised three times for 30 seconds

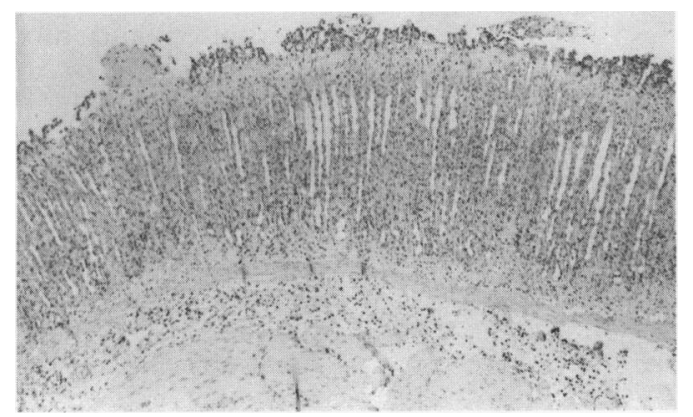

Figure 2: Histological section from the stomach of a rat treated for 48 hours with iodoacetamide $(0.1 \%)$ added to the drinking water. Note the superficial ulcerations of the mucosa with a mild inflammatory cell infiltrate. Haematoxylin and eosin $\times 73$.

\section{Determination of NOS activity}

NOS activity was monitored by the conversion of $\left[{ }^{3} \mathrm{H}\right]$-L-arginine to citrulline according to Bush et al. ${ }^{11}$ Mucosal scrapings (100 mg) were homogenised for 30 seconds at $4^{\circ} \mathrm{C}$ with a polytron (Kinematica $\mathrm{GmbH}$, KriensLuzern, Switzerland) in $0.3 \mathrm{ml}$ of ice cold $50 \mathrm{mM}$ TRIS $\mathrm{HCl}, \mathrm{pH} 7 \cdot 4$ containing $0.1 \mathrm{mM}$ EDTA, $0.1 \mathrm{mM}$ EGTA, $0.5 \mathrm{mM}$ dithiothreitol, $1 \mathrm{mM}$ phenylmethylsulphonyl fluoride. Homogenates were centrifuged at $20000 \times g$ for 60 minutes at $4^{\circ} \mathrm{C}$ and the supernatant was used as the source of NOS. Enzymatic reactions were conducted at $37^{\circ} \mathrm{C}$ in $50 \mathrm{mM}$ TRIS $\mathrm{HCl}, \mathrm{pH} 7 \cdot 4$ containing $100 \mu \mathrm{M}$ L-arginine, $100 \mu \mathrm{M}$ NADPH, $2 \mathrm{mM} \mathrm{CaCl}_{2}, 0.2-0.4 \mathrm{mg}$ supernatant proteins, and approximately $200000 \mathrm{dpm}$ of $\mathrm{L}-\left[2,3,4,5-{ }^{3} \mathrm{H}\right]$-arginine $\mathrm{HCl}(77 \mathrm{Ci} / \mathrm{mmol}$; Amersham, UK) to a final volume of $100 \mu \mathrm{l}$. In several experiments homogenates prepared from mucosal scrapings obtained from iodoacetamide treated and normal rats were incubated in the absence of $\mathrm{Ca}^{++}$or in the presence of 1-100 mM L-NAME, $10 \mathrm{mM}$ aminoguanidine or $60 \mathrm{mM}$ valine in the assay mixture. Enzymatic reactions were terminated by addition of $2.0 \mathrm{ml}$ of ice cold 'stop buffer': $20 \mathrm{mM}$ sodium acetate, $\mathrm{pH} 5 \cdot 5,1 \mathrm{mM}$ L-citulline, $2 \mathrm{mM}$ EDTA, and $0.2 \mathrm{mM}$ EDTA. Citrulline was determined by applying the samples $(2.0 \mathrm{ml})$ prepared as described above, to columns ( $1 \mathrm{~cm}$ diameter) containing $1 \mathrm{ml}$ of Dowex AG50W-X8, Na form, that had been pre-equilibrated with stop buffer. Columns were eluted with $4 \times 1.0 \mathrm{ml}$ of water collected into scintillation vials. Opti-fluor $(10 \mathrm{ml})$ was added to each vial and samples were counted in a Packard Tri-Carb liquid scintillation spectrometer. Citrulline was recovered in the 
Effect of TEMPOL on iodoacetamide induced gastritis

\begin{tabular}{lcccc}
\hline Treatment & $L A$ & \multicolumn{3}{c}{$I A+T E M P O L$} \\
\hline Days of treatment & 2 & 7 & 2 & 7 \\
Number & 10 & $9-19$ & 5 & 10 \\
Lesions (mm $2 / \mathrm{rat})$ & $27 \cdot 0(2 \cdot 2)$ & $71 \cdot 0(14 \cdot 7)$ & $36 \cdot 8(9 \cdot 0)$ & $22 \cdot 8(3 \cdot 2)^{\star}$ \\
MPO (U/g) & $2 \cdot 5(0 \cdot 4)$ & $1 \cdot 8(0 \cdot 2)$ & $1 \cdot 0(0 \cdot 2)$ & $1 \cdot 6(0 \cdot 3)$ \\
NOS (nmol/g/min) & $3 \cdot 2(0 \cdot 4)$ & $4 \cdot 2(0 \cdot 4)$ & $4 \cdot 2(0 \cdot 1)$ & $4 \cdot 0(0 \cdot 1)$ \\
\hline
\end{tabular}

Rats were treated with iodoacetamide $(0.1 \%)$ added to the drinking water with or without daily intragastric treatment with TEMPOL $(50 \mathrm{mg} / 100 \mathrm{~g})$. Rats were killed after two and seven days, the stomach removed, lesion area assessed, and mucosal MPO and NOS activities determined, as described in Methods. Results are mean (SEM). IA =iodoacetamide. ${ }^{\star}$ Significantly different from rats treated with iodoacetamide, $p<0.05$.

first $4.0 \mathrm{ml}$ of the Dowex column eluate to the extent of $96 \pm 2 \%$.

\section{Determination of eicosanoid generation}

One hundred and fifty mg of mucosa were placed in preweighed tubes containing $1.0 \mathrm{ml}$ of phosphate buffer $(50 \mathrm{mM}, \mathrm{pH} 7 \cdot 4)$. The mucosa was minced with scissors and centrifuged in an Eppendorf centrifuge for 10 seconds. The pellet was resuspended in 1.0 $\mathrm{ml}$ of the above buffer, incubated for one minute in a vortex mixer, $10 \mu \mathrm{g}$ indomethacin added, and the tubes centrifuged for 60 seconds. The supernatants were kept at $-20^{\circ} \mathrm{C}$ until radioimmunoassays were performed. The capability of the mucosa to generate leukotriene $\mathrm{B}_{4}\left(\mathrm{LTB}_{4}\right)$ and leukotriene $\mathrm{C}_{4}\left(\mathrm{LTC}_{4}\right)$ was expressed as $\mathrm{ng} / \mathrm{g}$ wet tissue weight.

\section{Measurement of $\mathrm{LTB}_{4}$}

$\mathrm{LTB}_{4}$ immunoreactivity was determined by a radioimmunoassay kit (Amersham, TRK 940). The assay combines the use of a high specific activity $\mathrm{LTB}_{4}$ tracer, an antiserum specific for $\mathrm{LTB}_{4}$ (cross reactivity $100 \%$ ), and a leukotriene standard (range 1.6 to 200 $\mathrm{pg} /$ tube). The specific binding of tracer is $42 \cdot 5 \%$, non-specific binding $2 \cdot 4 \%$. Fifty per cent $\mathrm{B} / \mathrm{Bo}$ displacement is obtained with $15 \mathrm{pg} /$ tube and $90 \% \mathrm{~B} / \mathrm{Bo}$ displacement with $2 \cdot 2 \mathrm{pg} /$ tube of $\mathrm{LTB}_{4}$.

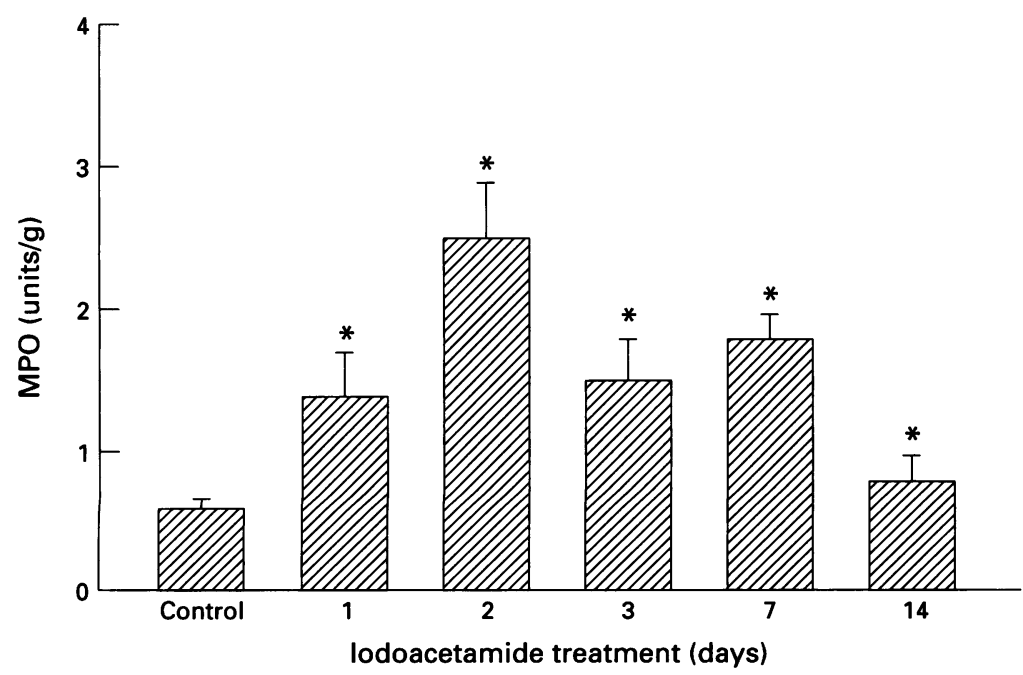

Figure 4: Gastric MPO activity in iodoacetamide treated rats. Rats were treated with iodoacetamide $(0.1 \%)$ added to the drinking water. Rats were killed after 1-14 days, the stomach was removed, washed, the mucosa scraped, and MPO activity determined, as described in Methods. Results are mean (SEM) of 4-19 rats in each time interval. * Significantly different from control, $p<0.05$.
Measurement of $L T C_{4}$

$\mathrm{LTC}_{4}$ immunoreactivity was determined by radioimmunoassay. The assay combines the use of a high specific activity $\mathrm{LTC}_{4}$ tritiated tracer with a monoclonal antibody specific for $\mathrm{LTC}_{4}$ and $\mathrm{LTC}_{4}$ standard (8-500 $\mathrm{pg} /$ tube). The assay uses highly specific $\mathrm{LTC}_{4}$ antiserum (cross reactivity $=100 \%$ ) and has low cross reactivity with leukotriene $\mathrm{D}_{4}\left(\mathrm{LTD}_{4}\right)(<5 \%)$. The specific binding of tracer is $40 \%-45 \%$ and non-specific binding is $1 \%-3 \%$. Fifty per cent $\mathrm{B} / \mathrm{Bo}$ displacement is obtained with 34 $\mathrm{pg} /$ tube, and $80 \% \mathrm{~B} /$ Bo displacement with 9.5 $\mathrm{pg} /$ tube of $\mathrm{LTC}_{4}$. The percentage coefficient of variation for within assay precision ranges from 7.68 (low) to 3.94 (high). The percentage coefficient of variation for the precision profile of the assay ranges from 1.64 to $3.49(n=9)$.

\section{Measurement of $P G E_{2}$}

$\mathrm{PGE}_{2}$ was determined by radioimmunoassay, as previously described. ${ }^{12}$

\section{Morphological studies}

Sections were obtained from four representative animals in each of the treatment groups. They were fixed in phosphate buffered formaldehyde, embedded in paraffin wax, and routine $5 \mu \mathrm{m}$ sections were prepared. Tissues were routinely stained with haematoxylin and eosin and were evaluated by light microscopy by a pathologist unaware of the experiments being performed.

\section{Statistical analysis}

Data are expressed as mean (SEM). Statistical analysis for significant differences was performed according to the Student's $t$ test for paired and unpaired data and the non-parametric Mann-Whitney U test.

\section{Results}

The addition of iodoacetamide $(0 \cdot 1 \%)$ to the drinking water induced gastric mucosal damage, which was apparent already after 24 hours and lasted for the entire 14 days of the experiment (Fig 1). At 24 and 48 hours the damage was observed adjacent to the fore-stomach. With prolongation of iodoacetamide treatment the damage affected the glandular part of the stomach. At 14 days lesions were widely distributed and most of the surface of the glandular part of the stomach was affected. Histologically, after the first $\mathbf{4 8}$ hours exposure to iodoacetamide the ulcerations were superficial and were accompanied by mild inflammatory cell infiltrate (Fig 2). After seven days of iodoacetamide treatment the ulcerations were deeper and affected half of the mucosal thickness (Fig 3). At 14 days the mucosal involvement was similar to that seen after seven days of treatment. Seven days after treatment with iodoacetamide and TEMPOL gastric lesions were significantly decreased by $68 \%$, compared with the lesions in rats treated with iodoacetamide only. The effective protection 


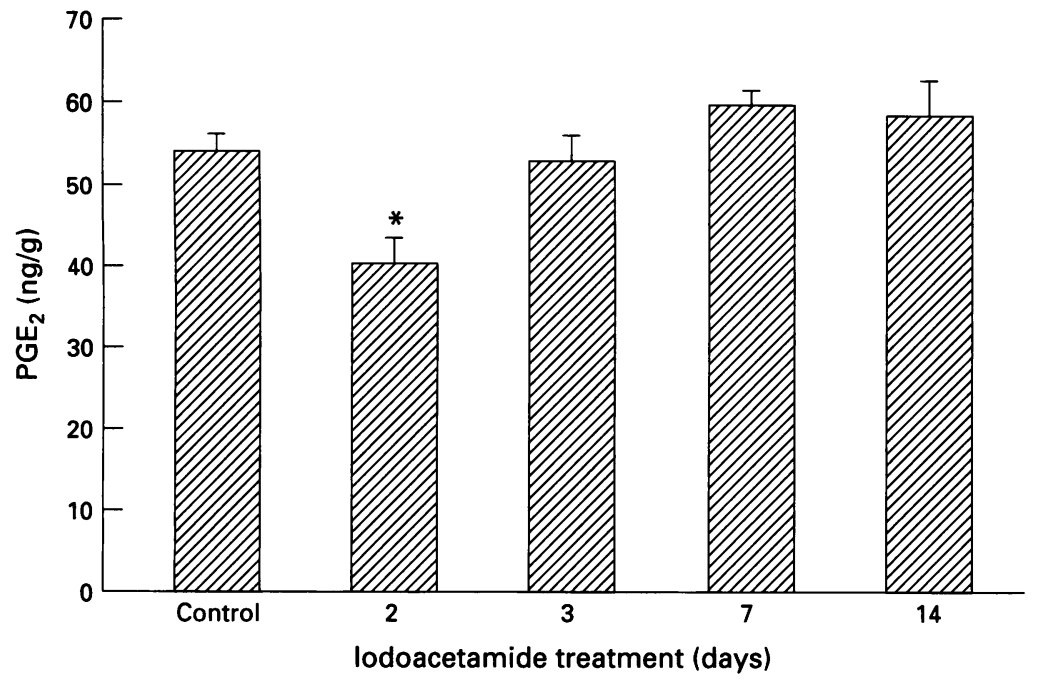

Figure 5: Gastric PGE generation in iodoacetamide treated rats. Rats were treated with iodoacetamide (0.1\%) added to the drinking water. Rats were killed after 1-14 days, the stomach was removed, washed, the mucosa scraped, and mucosal PGE 2 generation was determined, as described in Methods. Results are mean (SEM) of 9-20 rats in each time interval. ${ }^{\star}$ Significantly different from control, $p<0.05$.

provided by TEMPOL was not accompanied by significant effect on gastric MPO or NOS activities (Table).

Gastric mucosal MPO activity was significantly increased during the first seven days of iodoacetamide treatment. At 14 days gastric MPO activity was not stimulated when compared with its activity in normal gastric mucosa (Fig 4).

Iodoacetamide treatment induced a significant $(\mathrm{p}<0.05) 25 \%$ decrease in mucosal $\mathrm{PGE}_{2}$ generation but only after 48 hours. At all other time intervals mucosal $\mathrm{PGE}_{2}$ generation was similar in iodoacetamide treated and control rats (Fig 5). Iodoacetamide induced a twofold and 3.5-fold increase in mucosal $\mathrm{LTC}_{4}$ generation after seven and 14 days of treatment but not beforehand. At all time intervals mucosal $\mathrm{LTB}_{4}$ generation was similar in iodoacetamide and control rats (Fig 6).

Gastric mucosal NOS activity in control rats was $5 \cdot 7(0 \cdot 2) \mathrm{nmol} / \mathrm{g} / \mathrm{min}$. At all time intervals after iodoacetamide treatment, gastric NOS

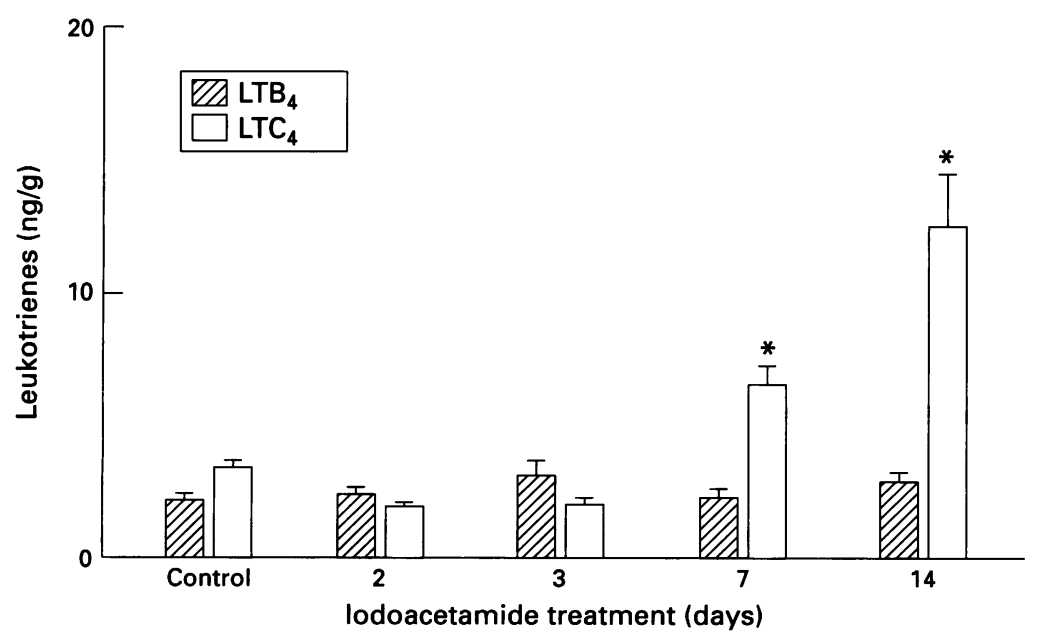

Figure 6: Gastric leukotriene generation in iodoacetamide treated rats. Rats were treated with iodoacetamide $(0 \cdot 1 \%)$ added to the drinking water. Rats were killed after 1-14 days, the stomach was removed, washed, the mucosa scraped, and mucosal LTB and LTC generation was determined, as described in Methods. Results are mean (SEM) of 8-16 rats

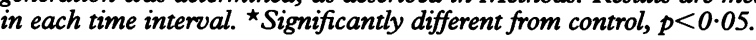

activity was significantly reduced when compared with its activity in normal rats (Fig 7). Both in control and in iodoacetamide treated rats deletion of calcium reduced gastric NOS activity by almost $50 \%$ and the addition of valine reduced the enzyme activity by $30 \%$. Aminoguanidine induced a $65.8(2.3) \%$ and $55.0(4 \cdot 0) \%$ decrease in the gastric enzyme activity of control and iodoacetamide treated rats, whereas in both groups L-NAME induced a dose dependent inhibition of the enzyme activity (Fig 8).

\section{Discussion}

In this study gastric damage induced by an $\mathrm{SH}$ blocker was found to be accompanied by decrease in gastric NOS activity, indicating the importance of both $\mathrm{SH}$ groups and NO to the maintenance of gastric mucosal integrity.

Endogenous SH compounds have an important role in maintaining mucosal integrity in the gastrointestinal tract. SH drugs prevent whereas SH blockers were shown to aggravate ethanol induced gastric injury. ${ }^{4} \mathrm{SH}$ blockers were also found to counteract the protective effect of prostaglandins against ethanol induced gastric damage. ${ }^{13}$ The $\mathrm{SH}$ blockers, iodoacetamide and ethylmaleimide, when added to the drinking water, were found to induce gastric mucosal injury, which was diminished by the sulphur containing amino acid, methionine. ${ }^{4}$ This study confirms the preliminary results reported by Szabo et al ${ }^{4}$ on the deleterious gastric effects of the $\mathrm{SH}$ alkylator, iodoacetamide, characterises in detail, the histological features of the model, the involvement of inflammatory mediators with specific evaluation of the involvement of NO in its pathogenesis. The results reported in this study also confirm the recent finding that iodoacetamide induces colonic damage and inflammation. ${ }^{5}$

Gastric injury induced by iodoacetamide was apparent within 24 hours and increased with time for up to 14 days. Mucosal ulceration with inflammatory cell infiltrate characterises the damage. After seven days of treatment the ulcers were deep, affected half of the mucosal thickness and, at 14 days, most of the gastric mucosa was affected. Usually, tissue injury is accompanied by increased generation of inflammatory mediators. It is of interest that, in iodoacetamide induced gastric injury $\mathrm{LTB}_{4}$ generation was not increased whereas $\mathrm{LTC}_{4}$ generation was increased, but only after seven and 14 days of treatment. It is, therefore, suggested that leukotriene contribution to the pathogenesis of tissue damage is limited and specific to each type of injury. We have previously shown that in iodoacetamide induced colitis mucosal leukotriene generation also did not correlate with the severity of tissue injury. ${ }^{5}$ Decrease in mucosal $\mathrm{PGE}_{2}$ generation could have also contributed to the pathogenesis of iodoacetamide induced damage. However, in this study only two days after iodoacetamide treatment gastric $\mathrm{PGE}_{2}$ generation was decreased and it is, therefore, unlikely that prostaglandins have an important role in 


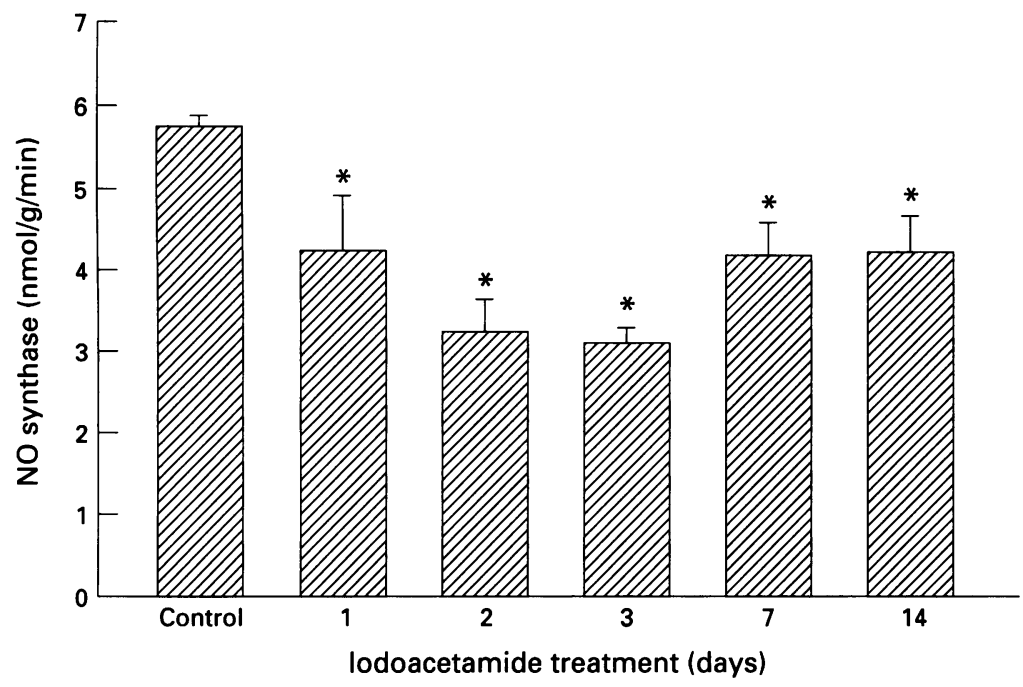

Figure 7: Gastric NOS activity in iodoacetamide treated rats. Rats were treated with iodoacetamide $(0 \cdot 1 \%)$ added to the drinking water. Rats were killed after 1-14 days, the stomach was removed, washed, gastric NOS activity was determined as described in Methods. Results are mean (SEM) of 7-16 rats in each time interval. * Significantly different from control, $p<0 \cdot 05$.

the pathogenesis of this type of tissue injury. In a similar fashion Hauser et al concluded that prostaglandins are minimally involved in the mechanism of gastric protection, whereas endogenous $\mathrm{SHs}$ are important mediators. ${ }^{14}$

In iodoacetamide induced gastritis mucosal MPO activity was found to be stimulated during the first seven days of treatment but not thereafter. The stimulated MPO activity may indicate the contribution of granulocytes to the inflammatory response at the beginning of the process. Similarly, in iodoacetamide induced colitis mucosal MPO activity was found to be stimulated also only during the first three days after damage induction. ${ }^{5}$ In a recent study the reliability of MPO as an indicator of neutrophil infiltration was questioned. ${ }^{15}$ However, it is also possible that during the second week of iodoacetamide treatment inflammatory cells

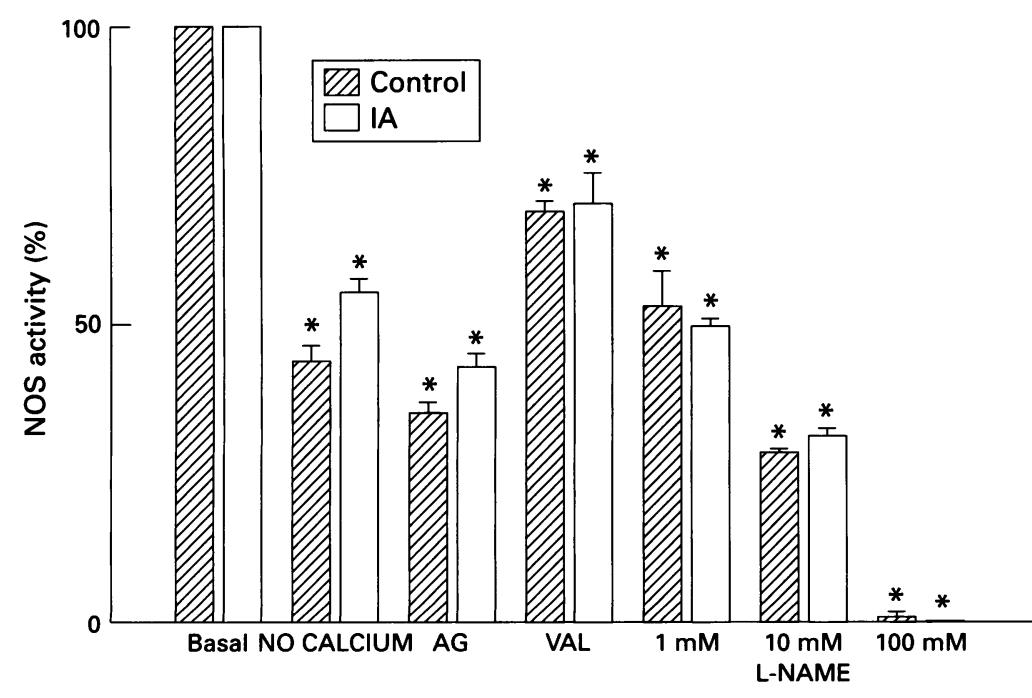

Figure 8: Effect of calcium sequestration, $L-N A M E$, aminoguanidine, and valine on gastric NOS activity. Gastric mucosal scrapings were obtained from normal rats and from rats treated for 72 hours with iodoacetamide $(0 \cdot 1 \%)$ added to their drinking water. NOS rats treated for 72 hours with iodoacetamide $(0 \cdot 1 \%)$ added to their drinking water. NOS $\mathrm{mM})$, valine $(60 \mathrm{mM})$ or aminoguanidine $(10 \mathrm{mM})$, as described in Methods. Basal activity in normal and iodoacetamide treated rats was $4.80(0.08)(n=4)$ and 2.59 $(0 \cdot 17)(n=4) \mathrm{nmol} / \mathrm{g} / \mathrm{min}$, respectively, and was regarded as $100 \%$. Results are mean (SEM) of determinations performed with tissue obtained from four rats in each group. $\star$ Significantly different from basal, $p<0.05$. other than granulocytes were responsible for the perpetuation of the inflammatory response.

In models of colonic inflammation increased $\mathrm{NO}_{\mathrm{x}}$ generation by stimulated NOS activity was reported ${ }^{16}$ and inhibition of colonic NOS activity was found to ameliorate the injury in several models of experimental colitis. ${ }^{17}$ In the model of iodoacetamide induced gastric injury herewith presented, gastric NOS activity was found to be decreased, showing the important contribution of $\mathrm{NO}$ to the maintenance of gastric mucosal integrity. In a similar way inhibition of gastric NOS activity was shown to amplify gastric damage induced by ethanol. ${ }^{6}$

Gastric NOS activity determined in this study by the conversion of L-arginine to citrulline is specific, as indicated by its effective inhibition by L-NAME. The comparatively small decrease in the conversion of L-arginine to citrulline in the presence of valine, which inhibits arginase activity ${ }^{18}$ rules out a significant contribution of arginase to the detected conversion of L-arginine to citrulline. Sequestration of calcium decreased by more than $50 \%$ gastric NOS activity in control rats and almost to the same extent also NOS activity in iodoacetamide treated rats. These results suggest that in the rat stomach at least half of the NOS activity represents the constitutive NOS isoform, which is calcium dependent. In the rat colon, on the other hand, only $17 \%$ of the total NOS activity is derived from the constitutive isoform. ${ }^{16}$ The relatively high representation of the constitutive NOS isoform in the stomach may suggest the importance of $\mathrm{NO}$ for the maintenance of gastric integrity and its vulnerability to reduction in its availability, such as that induced by L-NAME.

It is possible that the decrease in gastric NOS activity after treatment with iodoacetamide is a mechanism contributing to the induction of tissue injury in addition to its alkylation of $\mathrm{SH}$ compounds. The different representation of the constitutive and inducible NOS isoforms in rat colonic and gastric mucosa may explain, in part, their different vulnerability to NO depletion due to inhibition of NOS activity. Gastric mucosa may be dependent on higher concentrations of NO usually furnished by the relatively high representation of the constitutive isoform, whereas the integrity of colonic mucosa is less dependent on NO. In experimental colitis the increased NO generation is mainly derived from stimulation of the inducible isoform and inhibition of its activity is, therefore, beneficial. ${ }^{16}$

Acute NO synthesis inhibition causes an immediate rise in arterial blood pressure because of widespread vascular constriction. ${ }^{19}$ The addition of L-NAME to the drinking water induced in rats an increase of $38 \mathrm{~mm} \mathrm{Hg}$ in the mean arterial blood pressure. ${ }^{17}$ This rise in mean arterial blood pressure, associated with significant reduction in mesenteric blood flow, ${ }^{19}$ may reduce the extent of injury, thereby reducing the formation of reactive oxygen radicals. In the colon inhibition of NO generation was found to protect against injury, however, this was shown to be not only because of 
its vascular effect. ${ }^{17}$ On the other hand, in the stomach NO regulation of the mucosal haemodynamics, including blood flow and haemoglobin oxygen saturation, was shown to be responsible for its important contribution to the maintenance of mucosal integrity. ${ }^{6}$ These aspects of NO deprivation may certainly contribute to the severity of the gastric injury induced by iodoacetamide. Inhibition of NO formation was shown to aggravate gastric mucosal injury induced by ethanol. Decrease in the availability of NO induces a decrease in the resting mucosal blood flow, resulting in tissue hypoxia. ${ }^{6}$ The cumulative data thus show that endogenous NO is an essential protective factor in the pathogenesis of gastric injury induced by agents such as ethanol and iodoacetamide.

TEMPOL, the potent free radical scavenger, was found to effectively decrease the severity of gastric mucosal injury induced by iodoacetamide. We have reported that TEMPOL is a powerful protective agent against injury induced by various other irritants, indicating the important contribution of free radicals to the pathogenesis of gastric injury. ${ }^{9}$ These results suggest that scavenging of free radicals counteracts the deleterious effects of NO depletion, which contributes to the increased free radical generation by the hypoxic tissue. TEMPOL was found to have no effect on gastric NOS activity. However, as TEMPOL removes free radicals, which rapidly interact with NO and lower its concentration, TEMPOL may indirectly induce an increase of tissue NO concentrations. Increase in tissue NO concentrations may be an additional mechanism to explain the protective effect of TEMPOL. Potent scavengers, like TEMPOL, may therefore be useful for prevention of gastric mucosal injury induced by any irritant and, especially, by those that induce generation of free radicals. The importance of $\mathrm{SH}$ compounds in counteracting the deleterious effect of free radicals is also indicated by $N$-ethylmaleimide enhancement of gastric mucosal cell injury induced by oxygen free radicals generated by the xanthine oxidasexanthine system, an effect which is reduced by glutathione and cystamine. ${ }^{20}$

In conclusion, the results reported in this study show the importance of $\mathrm{SH}$ compounds and of NO to the maintenance of gastric mucosal integrity. NO donation, scavenging of free radicals, and provision of $\mathrm{SH}$ compounds may, therefore, be useful for prevention of gastric injury.

The authors are grateful to Dr S Szabo, who inspired the study.

Meister A, Anderson ME. Glutathione. Ann Rev Biochem 1983; 52: 711-60.

Olson CE. Glutathione modulates toxic oxygen metabolite injury of canine chief cell monolayers in primary culture. Am F Physiol 1984; 254: G49-56.

3 Wong HM, Tepperman BL. Reduced glutathione modulates $\mathrm{Ca}^{2+}$-mediated damage to rabbit isolated gastric mucosal cells. Am f Physiol 1994; 267: G1-9.

4 Szabo S, Trier JS, Brown A, Schnoor J. Sulphydryl blockers induce severe inflammatory gastritis in rats [Abstract]. Gastroenterology 1984; 86: 1271 .

5 Rachmilewitz D, Karmeli F, Okon E. Sulphydryl blocker colonic inflammation is ameliorated by inhibition of nitric oxide synthase activity. Gastroenterology 1995; 109: 98-106.

6 Masuda E, Kawano S, Nagano K, Tsuji S, Takei Y, Tsuji $\mathrm{M}$, et al. Endogenous nitric oxide modulates ethanolinduced gastric mucosal injury in rats. Gastroenterology 1995; 108: 58-64.

7 Pihan G, Regilo C, Szabo S. Free radicals and lipid peroxidation in ethanol- or aspirin-induced gastric mucosa injury. Dig Dis Sci 1987; 32: 1395-401.

8 Vaananen PM, Meddings JB, Wallace JL. Role of oxygenderived free radicals in indomethacin-induced gastric injury. Am f Physiol 1991; 261: G470-5.

9 Rachmilewitz D, Karmeli F, Okon E, Samuni A. A novel antiulcerogenic stable radical prevents gastric mucosal lesions in rats. Gut 1994; 35: 1181-8.

10 Bradley PP, Priebat DA, Christensen RD, Rothstein G. Measurement of cutaneous inflammation: estimation of neutrophil content with an enzyme marker. F Invest neutrophil content with
Dermatol 1982; 78: 206-9.

11 Bush PA, Gonzalez NE, Griscavage JM, Ignarro JL. Nitric oxide synthase from cerebellum catalyzes the formation of equimolar quantities of nitric oxide and citrulline from Larginine. Biochem Biophys Res Commun 1992; 185: 960-6.

12 Sharon P, Ligumsky M, Rachmilewitz D, Zor U. Role of prostaglandins in ulcerative colitis: enhanced production during active disease and inhibition by sulphasalazine. Gastroenterology 1978; 65: 638-40.

13 Szabo S, Trier JS, Frankel PW. Sulphydryl compounds may mediate gastric cytoprotection. Science 1981; 214: may m.

14 Hauser J, Szabo S. Extremely long protection by pyrazole derivatives against chemically induced gastric mucosal injury. F Pharmacol Exp Ther 1991; 256: 592-8.

15 Yamada T, Marshall S, Specian RD, Grisham MB. A comparative analysis of two models of colitis in rats. Gastroenterology 1992; 102: 1524-34.

16 Rachmilewitz D, Stamler JS, Bachwich D, Karmeli F, Ackerman Z, Loscalzo J, et al. Enhanced colonic nitric oxide generation and stimulated nitric oxide synthase activity in experimental colitis and in active inflammatory bowel disease. Gastroenterology 1993; 104: A766.

17 Rachmilewitz D, Karmeli F, Okon E, Bursztyn $M$. Experimental colitis is ameliorated by inhibition of nitric Experimental colitis is ameliorated by inhibition

18 Salter M, Knowels RG, Moncada S. Widespread tissue distribution, species distribution and changes in activity of $\mathrm{Ca}^{2+}$-dependent and $\mathrm{Ca}^{2+}$-independent nitric oxide synthases. FEBS Lett 1991; 291: 145-9.

19 Gardiner SM, Compton AM, Bennett T, Palmer BMJ, Moncada S. Control of regional blood flow by endothelium derived nitric oxide. Hypertension 1990; 15: 486-92.

$20 \mathrm{Li} \mathrm{T}$, Zhang XJ. Role of sulphydryl compounds in the oxygen radical induced injury of isolated gastric mucosal cells. Sheng Li fsueh Pao 1992; 44: 386-93. 